\title{
Functional Significance of SIGLECs Family in HCC and its Predictive Role in HCC Patients Undergoing Sorafenib
}

\section{Senbang Yao}

The Second Affiliated Hospital of Anhui Medical University

\section{Wenjun Chen}

Anhui Chest Hospital

\section{Tingting Chen}

The Second Affiliated Hospital of Anhui Medical University

He Zuo

The Second Affiliated Hospital of Anhui Medical University

\section{Ziran Bi}

The Second Affiliated Hospital of Anhui Medical University

\section{Xiuqing Zhang}

The Second Affiliated Hospital of Anhui Medical University

\section{Lulian Pang}

The Second Affiliated Hospital of Anhui Medical University

\section{Yanyan Jing}

The Second Affiliated Hospital of Anhui Medical University

\section{Xiangxiang Yin}

The Second Affiliated Hospital of Anhui Medical University Huaidong Cheng ( $\square$ chd1975ay@126.com )

The Second Affiliated Hospital of Anhui Medical University

\section{Research Article}

Keywords: Hepatocellular carcinoma, Sorafenib, Prognostic markers, Immunotherapy, Tumor progressio

Posted Date: September 7th, 2021

DOl: https://doi.org/10.21203/rs.3.rs-870519/v1

License: (c) (1) This work is licensed under a Creative Commons Attribution 4.0 International License. Read Full License 


\title{
Functional significance of SIGLECs family in HCC and its predictive role in $\mathrm{HCC}$ patients Undergoing sorafenib
}

\author{
Senbang Yao ${ }^{\dagger 1,2}$, Wenjun Chen ${ }^{\dagger 2,3}$, Tingting Chen $^{\dagger 1}$, He Zuo ${ }^{1,2}$, Ziran Bi ${ }^{1,2}$, Xiuqing Zhang ${ }^{1,2}$, \\ Lulian Pang ${ }^{1,2}$, Yanyan Jing ${ }^{1,2}$, Xiangxiang Yin ${ }^{1,2}$ and Huaidong Cheng ${ }^{1,2}$ \\ ${ }^{1}$ Department of Oncology, The Second Affiliated Hospital of Anhui Medical University, Hefei, \\ Anhui, China; \\ ${ }^{2}$ Department of Oncology, Anhui Medical University, Hefei, Anhui, China \\ ${ }^{3}$ Department of Oncology, Anhui Chest Hospital, Hefei, Anhui, China
}

$\dagger$ These authors contributed equally to this work.

\section{*Correspondence to:}

Huaidong Cheng, Department of Oncology, The Second Affiliated Hospital of Anhui Medical University, Anhui Medical University, 678 Furong Road, Hefei, Anhui 230601, China.

Email: chd1975ay@126.com

\section{Abstract}

Background and Aims: Hepatocellular carcinoma (HCC) is an aggressively malignant type of cancer with a complex pathogenesis. For HCC patients, there is not only a deficiency of valuable therapeutic targets but also a deficiency of prognostic biomarkers. Although some progress has been made in the immunotherapy of HCC, sorafenib remains one of the irreplaceable therapeutic for advanced HCC patients. However, after 10 years of exploration on sorafenib clinical application, there are still no confirmed prognostic factors of therapeutic effect. Methods: The clinical significance and molecular work of the SIGLEC family were assessed by comprehensive bioinformatic analysis.The TCGA, GEO and HCCDB databases were used to explore the expression of SIGLEC family genes in HCC. The Kaplan-Meier Plotter database was analysed to discover the connection between SIGLEC family genes and prognosis of patients. Besides, possible connections between differentially expressed genes (DEGs) of SIGLEC family and tumor-associated immunity were evaluated using TIMER. Results: The outcomes showed that the mRNA levels of most SIGLEC family genes were significantly down-regulated in HCC compared with normal tissues. Low protein and mRNA expression of SIGLECs were found to be considerably correlated with tumor grade and clinical cancer stages in HCC patients. Tumor related SIGLECs family genes were associated with tumor immune infiltrating cell. To our surprise, the high expression of SIGLECs family is significantly related with the better prognosis of HCC patients treated with sorafenib.

Conclusions: In conclusion, SIGLEC family genes have considerable prognostic value in HCC and they may play main roles in regulating cancer progress and the immune cell infiltration. More importantly, we revealed that SIGLEC family genes may become a predictive index for the efficacy evaluation of sorafenib someday. 


\section{Keywords}

Hepatocellular carcinoma; Sorafenib; Prognostic markers; Immunotherapy; Tumor progression.

\section{Introduction}

Malignant tumors pose a severe threat to the health of people all over the globe, and many people's lives are taken away by Hepatocellular carcinoma (HCC) under the action of pathogenic factors such as hepatitis and alcohol. ${ }^{1}$ HCC is the sixth most widespread cancer and the third leading reason of cancer mortality today. ${ }^{2}$ Although some patients with liver cancer are cured by local hepatectomy, the overall survival outcome of liver cancer is still poor. The bad prognosis of HCC can be attributed to the reason that diagnosis is usually made at a late phase in cancer development. ${ }^{3}$ Sorafenib, a multi-targeted tyrosine kinase inhibitor, for the treatment of advanced-stage HCC patients in 2007 highlights the possible of such therapies in treating this malignant cancer. ${ }^{4}$ But the median lifetime expectancy of HCC patients on sorafenib is just 1 year. However, after 10 years of exploration on sorafenib clinical application, there are still no confirmed prognostic factors of therapeutic effect. ${ }^{5-7}$

Siglecs are a family of sialic acid-recognition proteins expressed primarily on leukocytes that have controlling effects in the fine-tuning of leukocyte activities. ${ }^{8}$ The role of Siglecs in neoplasm has already been described, and therapeutic agents targeting Siglecs are being investigated. ${ }^{9}$ Siglecs are mainly expressed on the external of immune cells.Thus, siglecs can became a target for moderating immunological proceedings. ${ }^{10}$ The association between Siglecs expression and prognosis of patients with tumor was evaluated by many scholars. Kensuke Yamada reported the connection between Siglec-7 and CRC prognosis. ${ }^{11}$ Wang et al identified Siglec-15 as a prospective target for tumor immunotherapy. ${ }^{12}$ Ye et al showed SIGLEC family genes may manipulate TME by chemokine axis, performing vital roles in cancer patients'prognosis. ${ }^{13}$ However, the biological function of SIGLEC family members in HCC remains uncertain, while a exhaustive exploring of biological and molecular process is essential to develop new treatment prognostic indicators and target spots.

In this research, we explored this question by detecting the protein and transcriptional expression of the SIGLECs family genes through TCGA, GEO and Human Protein Atlas (HPA) database. Then we used multi-dimensional analysis, we structured functional networks and genomic alterations associated to SIGLECs in HCC and explored its effect in tumor immunity. In addition, we also analyzed the clinical characteristics and prognostic value of SIGLECs family genes in HCC. This study reveals the biological meaning and prognostic importance of SIGLECs in HCC, which will be advantageous to the therapy and diagnosis of HCC. We surprisingly found that SIGLECs can be used as an effective prognostic marker of sorafenib in the treatment of HCC, which is the most important clinical significance of this study.

\section{Methods}

\section{Differential expression of SIGLECs at transcriptional level}

The Cancer Genome Atlas (TCGA) is a well-known cancer genomics project. It collects genomic information of more than 20000 primary cancers and matches 33 normal samples of cancer types. ${ }^{14}$ The Gene Expression Profiling Interactive Analysis (GEPIA) is an database that includes 9,736 tumor samples and 8,587 normal samples from TCGA and the GTEx projects. We 
used GEPIA to explore the difference of SIGLECs expression between cancer tissues and corresponding normal tissues in TCGA data. ${ }^{15}$ UALCAN is a extensive, usable, and coactive web resource for analyzing cancer genomic information. It provides easy entrance to publicly available oncology data, such as TCGA, MET500 and CPTAC. ${ }^{16}$ We used UALCAN to correlate the members of SIGLECs family with tumor staging, and grading in HCC patients..

GEO is a public genome data storage center where MIAME-compliant data can be uploaded. Biological and medical researchers can query and download related gene expression profiles ${ }^{17}$. GSE $14520^{18}$ and GSE22058 ${ }^{19}$ datasets were obtained from the GEO database. In addition, International Cancer Genome Consortium (ICGC) was also used to explore the differential expression of SIGLECs at transcriptional leve. The specific data set is ICGC-LIRI-JP, which contains 231 liver cancer samples, RNA-seq data and clinical information. ${ }^{20}$

\section{Differential expression of SIGLECs at protein level}

In order to study the expression of SIGLECs in HCC at protein level, The Human Protein Atlas

(HPA) was used to directly observe the immunohistochemical pictures of SIGLECs family proteins in HCC and normal specimens. ${ }^{21}$ The protein expression of SIGLECs in normal liver tissue exists in the tissue module, which contains 44 kinds of normal human tissue protein expression data derived from the antibody-based protein profiling using immunohistochemistry.

\section{Functional clustering and Molecular Network Construction of SIGLECs in HCC}

GeneMANIA is a website interface for generating hypotheses about gene function, analyzing gene lists and prioritizing genes for functional assays..$^{22}$ In our study, we submitted members of the SIGLECs family to GeneMANIA, to clarify the functional connection network between SIGLECs and its associated genes. Specific network categories include: shared protein domains,co-expression,physical interactions,predicted,co-localization and genetic interactions.

WebGestalt (WEB-based Gene SeT AnaLysis Toolkit) is a functional enrichment analysis instrument. Pathways and GO functions of SIGELCs and their correlated genes were enriched by WebGestalt. The GO functional enrichment include the cellular component (CC), biological process (BP), molecular function (MF) and the KEGG pathway was showed by analysis of pathway. Metascape is a web-based entrance used to provide a inclusive gene list annotation and analysis source for investigational biologists. We analyzed the gene enrichment of SIGLECs family again by DisGeNET in Metascape.

\section{Tumor immunology analysis}

Tumor immunology was estimated using TIMER (Tumor IMmune Estimation Resource) ${ }^{23}$, which is a website used for comprehensive analysis of Tumor-Infiltrating Immune Cells. The immune infiltration estimation of SIGLECs was performed in LIHC by TIMER. We explored SIGLECs expression in LIHC and the correlation of SIGLECs expression with the abundance of immune infiltrates, including CD4 $+\mathrm{T}$ cells, B cells, and CD8 $+\mathrm{T}$ cells. And we showed the purity-corrected partial Spearman's rho value and statistical significance by drawing the Three-line table. Based on TIMER, we also investigated the correlation between SIGLECs family members expression and PD1, PD-L1 and CTLA4. 
cBioPortal is an network platform based on TCGA data that integrates data mining, data integration and visualization ${ }^{24}$. An overview of genetic alterations per sample in SIGLECs was displayed in OncoPrint. It was used to analyze SIGLECs alterations in the TCGA LIHC patients. The search parameters included mutation, CNVs, mRNA expression, and survival.

In our research, the prognostic function of mRNA expression of SIGLECs famliy genes in HCC was analyzed by The Kaplan Meier plotter, which is used to assess the effect of various genes (mRNA, miRNA, protein) on survival in multiple cancer types. Sources for the databases include TCGA, EGA, and GEO.The two patient cohorts are contrasted by a Kaplan-Meier survival analysis, and the HR with $95 \%$ confidence intervals and logrank $\mathrm{P}$ value are assessed. Databases and clinical information are supervised regularly. ${ }^{25}$

\section{Statistical analysis}

The expression of SIGLECs was analysed by using T-test. Kaplan-Meier analysis were used to evaluate the prognostic differences. The logrank $P<0.05$ reveals the significance of prognosis differences. We used Wilcoxon signed-rank test, One-way ANOVA test, and logistic regression to explore relationships among clinicalpathologic features and SIGLECs expression. The connection between SIGLECs and immune sign score or gene expression levels was calculated by using the Spearman method. Statistical analysis and figures were generated by R(v.3.6.3). Some plots were produced using the graphics package ggplot2 (https://ggplot2.tidyverse.org)

\section{Results}

\section{The Low mRNA Expression of SIGLECs Family Genes in Patients With HCC}

The analysis process of this study is shown in Fig. 1.

For purpose of study the expression of SIGLECs family members in patients with HCC, we used ICGC and GEO database to analyze the expression differences between cancer tissues and corresponding normal tissues. First, ICGC-LIRI-JP datasets revealed that SIGLECs family members were significantly down-regulated in HCC tissues compared with adjacent non-tumour tissues (Fig. 2A) . Furthermore, based on GSE22058 dataset, SIGLECs family members were found to be down-regulated in $\mathrm{HCC}$ tissues compared with that in the paired adjacent non-tumour tissues (Fig. 2B). Similar results were also shown in the GSE14520 (Fig. 2C). These results mean that mRNA expressions of SIGLECs family genes were significantly lower in HCC tissues. The heat map shows that the most differentially expressed genes include SIGLEC1 and SIGLEC7 (Fig. 2D ) . SIGLECs family members showed decreased expression in cancer tissues in different HCCDB data, and there was significant difference. The comprehensive information of the data sets is summarized in Table 1.

Then we explored the mRNA expression of SIGLECs family members through the TCGA data. The mRNA expression of most SIGLECs family genes in HCC tissues were significantly down-regulated compared with normal samples, which was similar to the results of GEO analysis (Fig. 3).

Table 1. The information of HCCDB datasets.

\begin{tabular}{llllll}
\hline & Dataset ID & P-value & No. of Adjacent & No. of HCC & Source \\
\hline SIGLEC1 & & & & \\
\hline
\end{tabular}




\begin{tabular}{|c|c|c|c|c|c|}
\hline & HCCDB1 & $2.340 \mathrm{e}-30$ & 97 & 100 & GSE22058 \\
\hline & HCCDB16 & 0.00000153 & 52 & 115 & GSE76427 \\
\hline \multicolumn{6}{|l|}{ SIGLEC2 } \\
\hline & HCCDB7 & 0.02824 & 82 & 80 & GSE10143 \\
\hline \multicolumn{6}{|l|}{ SIGLEC3 } \\
\hline & HCCDB18 & 0.004231 & 177 & 212 & ICGC-LIRI-JP \\
\hline \multicolumn{6}{|l|}{ SIGLEC4 } \\
\hline & HCCDB15 & 0.00000282 & 49 & 356 & TCGA-LIHC \\
\hline \multicolumn{6}{|l|}{ SIGLEC5 } \\
\hline & HCCDB13 & 0.0002155 & 168 & 228 & GSE63898 \\
\hline \multicolumn{6}{|l|}{ SIGLEC6 } \\
\hline & HCCDB6 & $1.890 \mathrm{e}-15$ & 220 & 225 & GSE14520 \\
\hline \multicolumn{6}{|l|}{ SIGLEC7 } \\
\hline & HCCDB1 & $2.430 \mathrm{e}-34$ & 97 & 100 & GSE22058 \\
\hline \multicolumn{6}{|l|}{ SIGLEC8 } \\
\hline & HCCDB15 & 0.0000017 & 49 & 356 & TCGA-LIHC \\
\hline \multicolumn{6}{|l|}{ SIGLEC9 } \\
\hline & HCCDB16 & 0.0008423 & 52 & 115 & GSE76427 \\
\hline \multicolumn{6}{|l|}{ SIGLEC10 } \\
\hline & HCCDB12 & 0.01251 & 80 & 81 & GSE54236 \\
\hline \multicolumn{6}{|l|}{ SIGLEC11 } \\
\hline & HCCDB4 & $5.760 \mathrm{e}-37$ & 193 & 240 & GSE36376 \\
\hline \multicolumn{6}{|l|}{ SIGLEC14 } \\
\hline & HCCDB13 & $3.540 \mathrm{e}-22$ & 168 & 228 & GSE63898 \\
\hline \multicolumn{6}{|l|}{ SIGLEC15 } \\
\hline & HCCDB6 & 0.07336 & 220 & 225 & GSE14520 \\
\hline \multicolumn{6}{|l|}{ SIGLEC16 } \\
\hline & HCCDB17 & 0.00000385 & 52 & 115 & GSE76427 \\
\hline
\end{tabular}




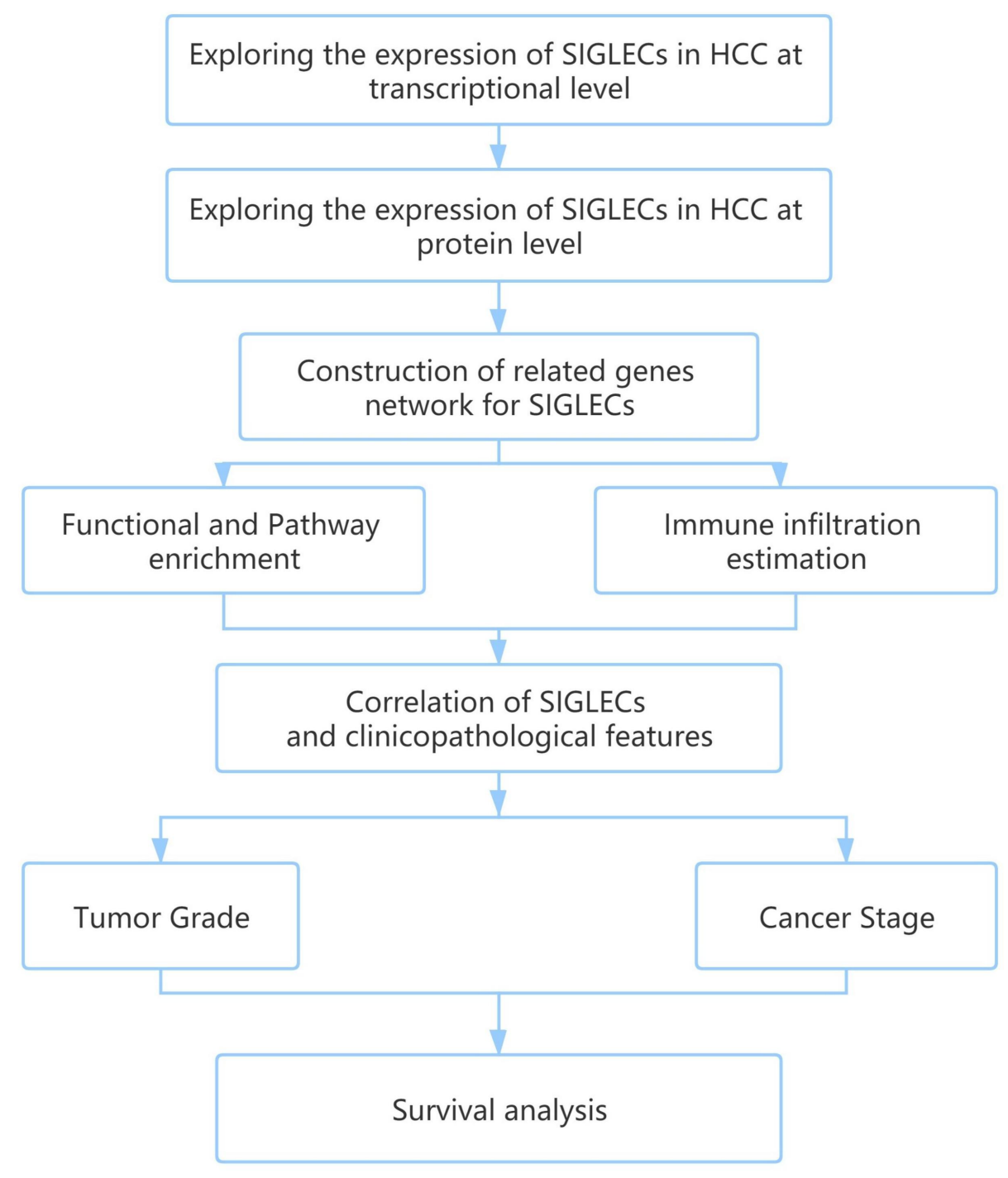

Fig. 1. The flow graph of analysis process in this study. 


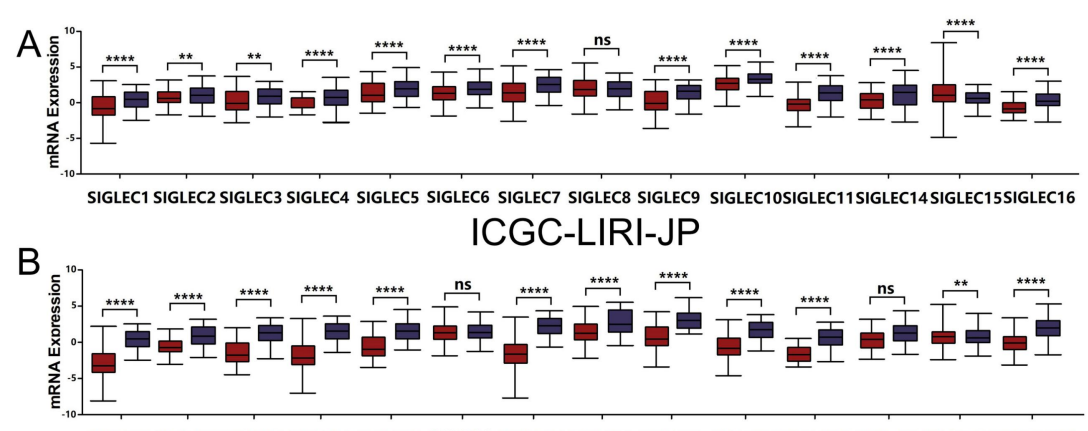

SIGLEC1 SIGLEC2 SIGLEC3 SIGLEC4 SIGLEC5 SIGLEC6 SIGLEC7 SIGLEC8 SIGLEC9 SIGLEC10SIGLEC11SIGLEC14SIGLEC15SIGLEC16 GSE22058

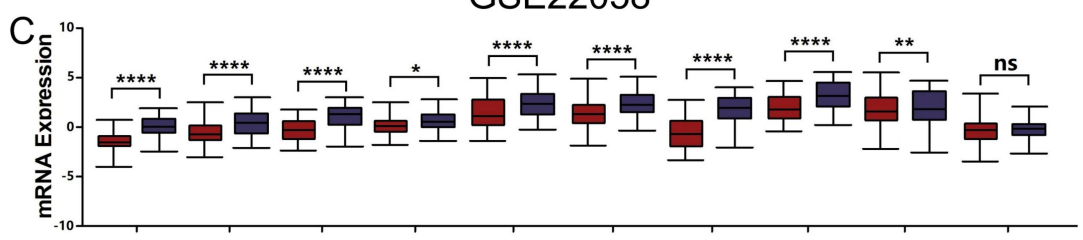

SIGLEC1 SIGLEC2 SIGLEC3 SIGLEC4 SIGLEC5 SIGLEC6 SIGLEC7 SIGLEC8 SIGLEC9 SIGLEC15 GSE14520

$\mathrm{D}$

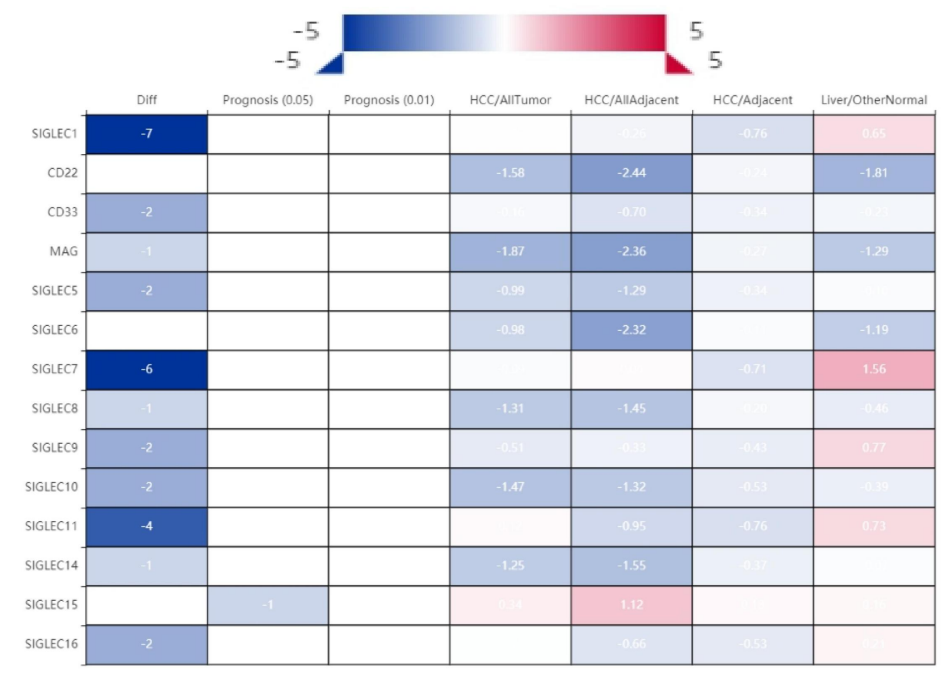

Fig. 2. Transcriptional expressions of SIGLECs family members in ICGC and GEO database. (A) Expression of SIGLECs family in cancer and normal tissues of ICGC database. (B) Expression of SIGLECs family in cancer and normal tissues of GEO database(GSE22058). (C) Expression of SIGLECs family in cancer and normal tissues of GEO database(GSE14520) (D) Heatmap representation of the differential expression. The Diff means the number of differentially expressed datasets, blue indicates down-expression, red indicates over-expression. 


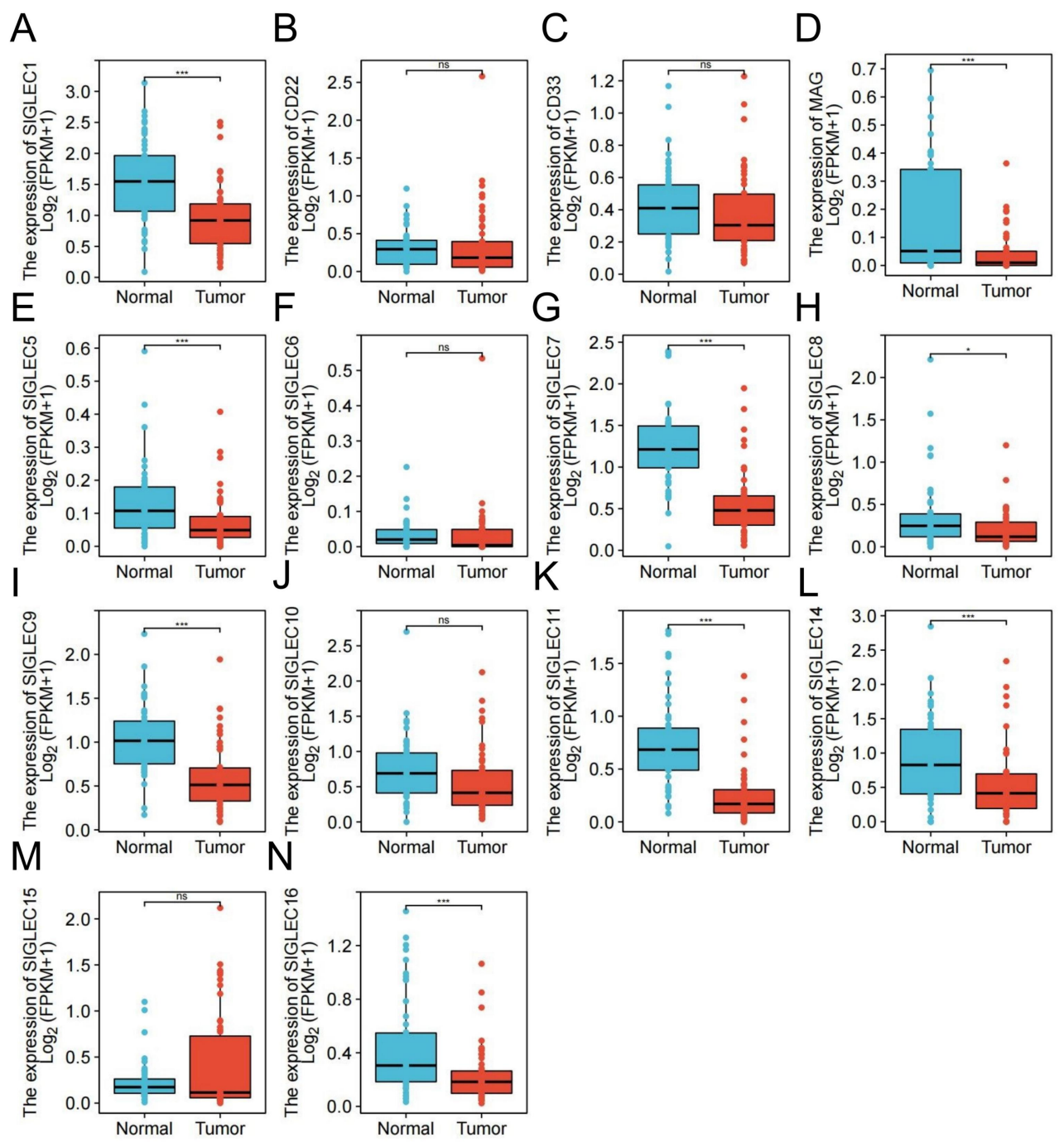

Fig. 3. Low mRNA expressions of SIGLECs family genes in HCC patients and normal liver tissues (TCGA). The mRNA expressions of most SIGLECs family genes were significantly down-regulated in HCC patients from the TCGA database (A-S). ${ }^{* * *} \mathrm{p}<0.001$.

\section{Difference of protein expression of SIGELCs family genes in HCC patients}

We investigated the protein expression of SIGLECs family genes in HCC by Human protein Atlas. Similar to the results of mRNA analysis, the expression of SIGLECs protein was low in HCC tissues detected by HPA (Fig. 4). Low protein expressions of SIGLEC3, SIGLEC4, SIGLEC5, SIGLEC8, SIGLEC9, and SIGLEC14 were exploreed in HCC tissues, while their relatively high expressions of protein were noticed in normal liver tissues. Negative protein expression of SIGLEC1, SIGLEC 2, SIGLEC6, SIGLEC10, and SIGLEC11 were observed both at normal liver tissues and HCC tissues (Fig. 4). Overall, our findings indicated that the expression of SIGLECs family genes was significantly lower in patients with HCC. 

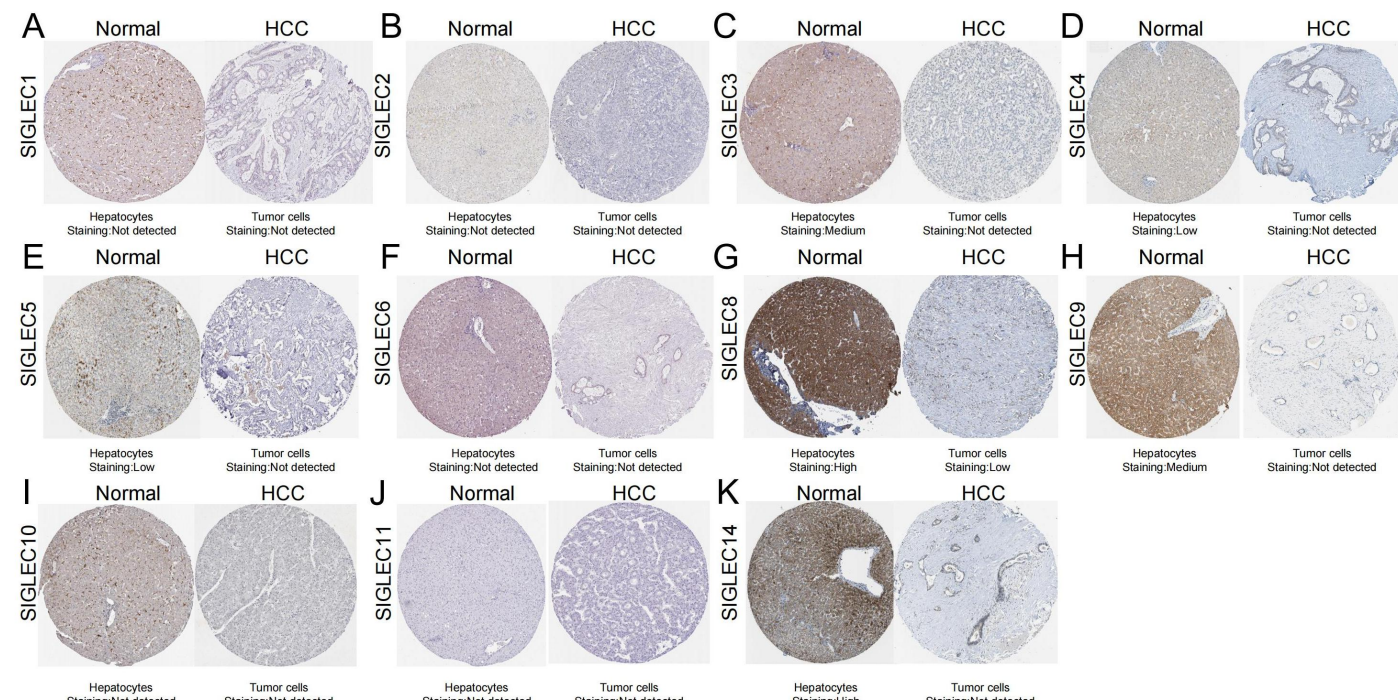

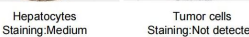

Fig. 4. Immunohistochemical images of SIGLECs family genes in normal liver tissues and HCC tissues (HPA database).

\section{Functional enrichment Analysis of SIGLECs Family genes in HCC}

The network of SIGLECs family genes and their related genes was constructed by GeneMANIA (Fig. 5A) and PINA (Fig. 5B). Through the functional network diagram, we can grasp the information of positive and negative related genes that interact with the SIGLECs family, and the specific information is indicated in the diagram.

We used WebGestalt to analyze the GO function and pathway of SIGLECs and its related genes.The biological processes such as cell adhesion, biological adhesion, neutrophil activation involved in immune response were significantly regulated by the SIGLECs in HCC (Fig. 5C). Cellular modules including tertiary granule membrane, tertiary granule, and secretory granule membrane (Fig. 5D). Besides,SIGLECs also remarkably influenced the molecular functions (Fig. $5 \mathrm{E}$ ), such as sialic acid binding, CD4 receptor binding and glycosphingolipid binding.

Through KEGG analysis, we found that the pathways involved in SIGELCs include Cell adhesion molecules (CAMs), Hematopoietic cell lineage, and B cell receptor signaling pathway (Fig. 5F). We carried out Gene list enrichment on the members of the SIGLECs family by Metascape. The results show that the SIGLECs family is obviously enriched in Tumor Immunity, Guillain-Barre Syndrome, IMMUNE SUPPRESSION and other functions(Fig. 5G). 
A
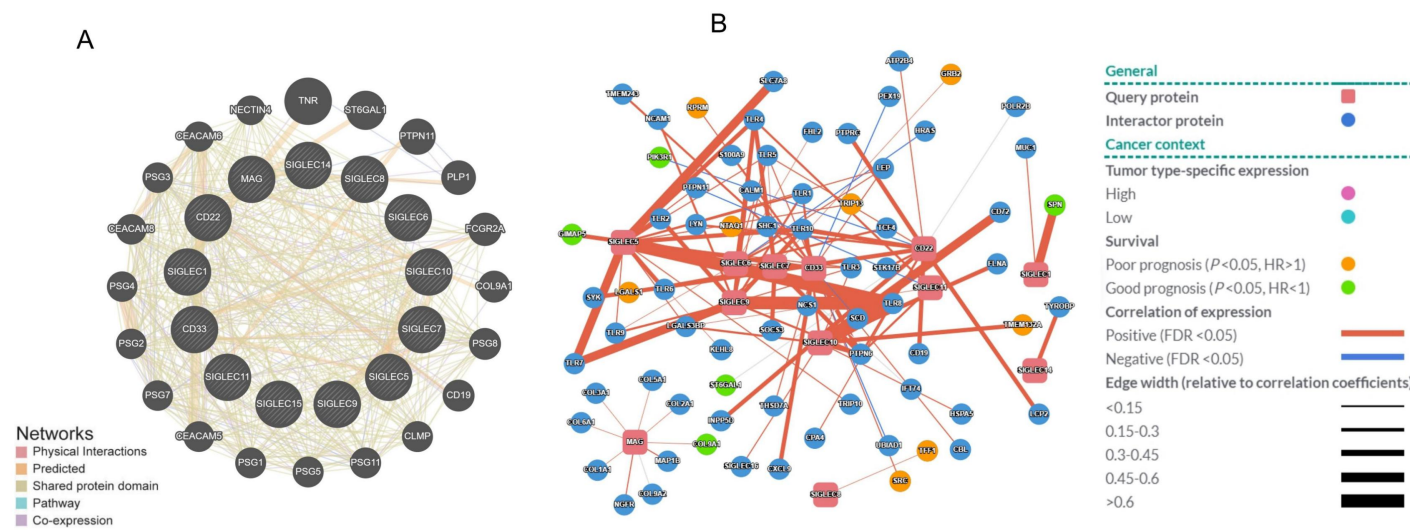

in Co-expression

C GO-Biological process

FDR $\leq 0.05 \quad$ FDR $>0.05$

D GO-Cellular component
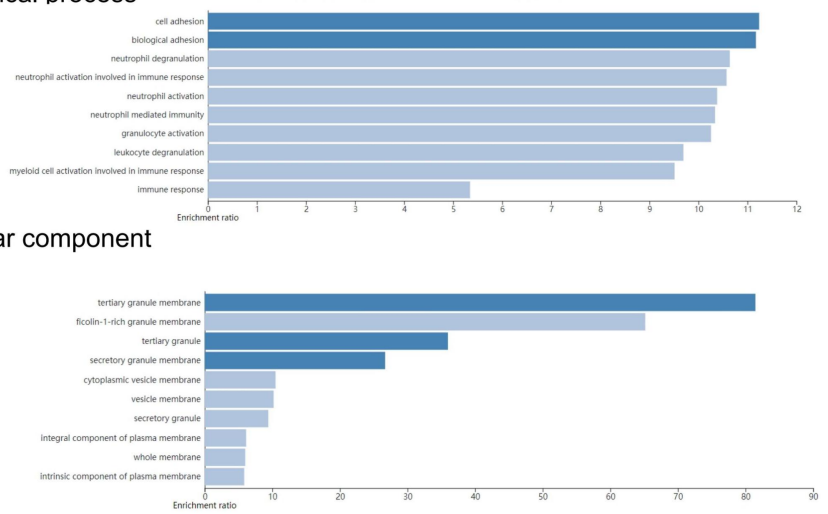

E GO-Molecular function

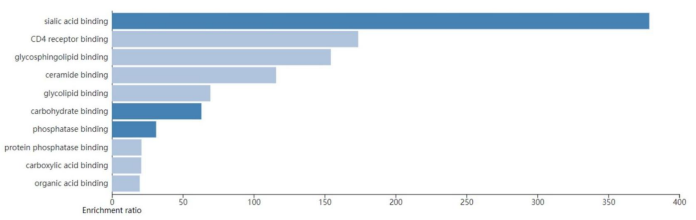

F KEGG pathway

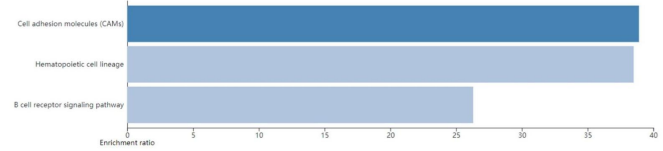

G

Summary of enrichment analysis in DisGeNET

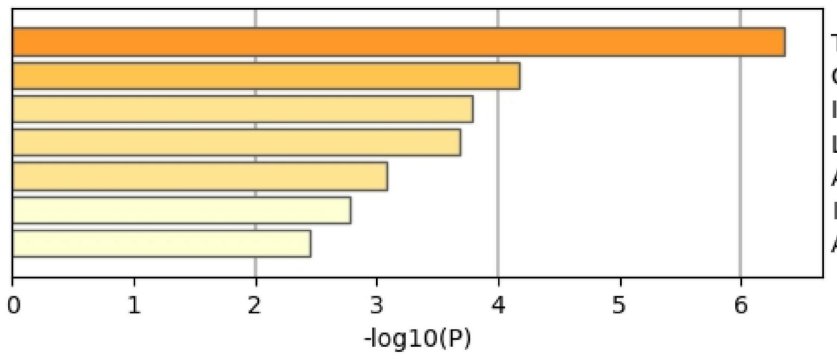

Tumor Immunity Guillain-Barre Syndrome IMMUNE SUPPRESSION Leukemia, B-Cell Alzheimer Disease, Late Onset Infection Acute leukemia

Fig. 5. Function enrichment of SIGLECs family genes in HCC. (A) Network of SIGLECs family genes and their related genes was evaluated by GeneMANIA. (B) Interaction Network Analysis of SIGLECs family members from PINA platform; (C) Cellular module; (D) Biological processes; (E) Molecular functions; (F) KEGG pathway analysis. (G) Gene list enrichment. 
Analysis of correlation between mRNA expression of SIGLECs family genes and HCC immune infiltration degree

TIMER database is used to explore the relationship between SIGLECs family members and HCC immune infiltration. The correlation of mRNA expression of most SIGLECs with B cell was statistically significant. While mRNA expression of SIGLEC1, SIGLEC2, SIGLEC3, SIGLEC5, SIGLEC6, SIGLEC7, SIGLEC8, SIGLEC9, SIGLEC10, SIGLEC11, SIGLEC14, SIGLEC15, and SIGLEC16 was obviously related to CD4+ T cell. In addition, mRNA expression of SIGLEC1, SIGleC2, SIGLEC3, SIGLEC5, SigleC6, SigleC7, SIGlEC8, SIGLEC9, SIGLEC10, SIGLEC11, SIGLEC14, and SIGLEC16 had remarkable connections with infiltrating levels of $\mathrm{CD} 8+\mathrm{T}$ cells in HCC. The following data are shown correlations between gene expressions of SIGLECs family and PD-1 (Fig. 6A), PD-L1(Fig. 6B) and CTLA-4 (Fig. 6C).

Table 2. Association between the different expressions of SIGLECs family with $T$ cell infiltration levels and B cell infiltration levels in HCC.

\begin{tabular}{|c|c|c|c|c|c|c|}
\hline \multirow[b]{2}{*}{ SIGLECs } & \multicolumn{2}{|c|}{ B Cell } & \multicolumn{2}{|c|}{ CD4+ T Cell } & \multicolumn{2}{|c|}{ CD8+ T Cell } \\
\hline & Cor & P-value & Cor & P-value & Cor & P-value \\
\hline SIGLEC1 & 0.458 & 3.29E-19 & 0.281 & $1.22 \mathrm{E}-07$ & 0.535 & $1.06 E-26$ \\
\hline SIGLEC2 & 0.337 & $1.45 E-10$ & 0.388 & 8.00E-14 & 0.325 & $7.85 \mathrm{E}-10$ \\
\hline SIGLEC3 & 0.561 & $6.30 \mathrm{E}-30$ & 0.332 & 2.55E-10 & 0.572 & $4.51 \mathrm{E}-31$ \\
\hline SIGLEC4 & 0.058 & $2.86 \mathrm{E}-01$ & 0.059 & $2.78 \mathrm{E}-01$ & 0.102 & 5.97E-01 \\
\hline SIGLEC5 & 0.550 & 1.28E-28 & 0.434 & 3.18E-17 & 0.513 & $2.47 E-24$ \\
\hline SIGLEC6 & 0.249 & 3.01E-06 & 0.279 & 1.51E-07 & 0.277 & $1.90 \mathrm{E}-07$ \\
\hline SIGLEC7 & 0.558 & $1.53 E-29$ & 0.313 & 2.78E-09 & 0.597 & $1.89 E-34$ \\
\hline SIGLEC8 & 0.335 & $1.70 \mathrm{E}-10$ & 0.409 & $2.56 \mathrm{E}-15$ & 0.387 & $1.18 E-13$ \\
\hline SIGLEC9 & 0.559 & 1.12E-29 & 0.412 & 1.64E-15 & 0.562 & $7.94 \mathrm{E}-30$ \\
\hline SIGLEC10 & 0.601 & 3.81E-35 & 0.512 & 2.11E-24 & 0.577 & $1.01 E-31$ \\
\hline SIGLEC11 & 0.251 & 2.37E-06 & 0.098 & 6.93E-02 & 0.363 & 4.02E-12 \\
\hline SIGLEC14 & 0.370 & $1.27 E-12$ & 0.220 & $3.88 \mathrm{E}-05$ & 0.402 & 1.11E-14 \\
\hline SIGLEC15 & 0.119 & $2.74 \mathrm{E}-02$ & 0.143 & $7.86 \mathrm{E}-03$ & 0.001 & $9.93 \mathrm{E}-01$ \\
\hline SIGLEC16 & 0.18 & 8.17E-04 & 0.122 & 2.37E-02 & 0.284 & 9.10E-08 \\
\hline
\end{tabular}




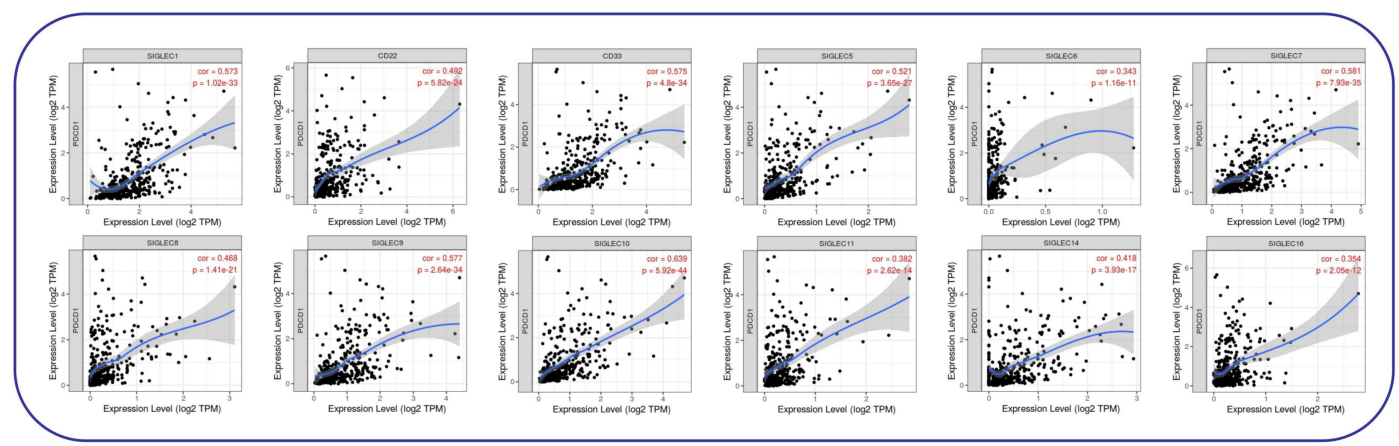

$\mathrm{B}$

SIGLECs VS PD-L1
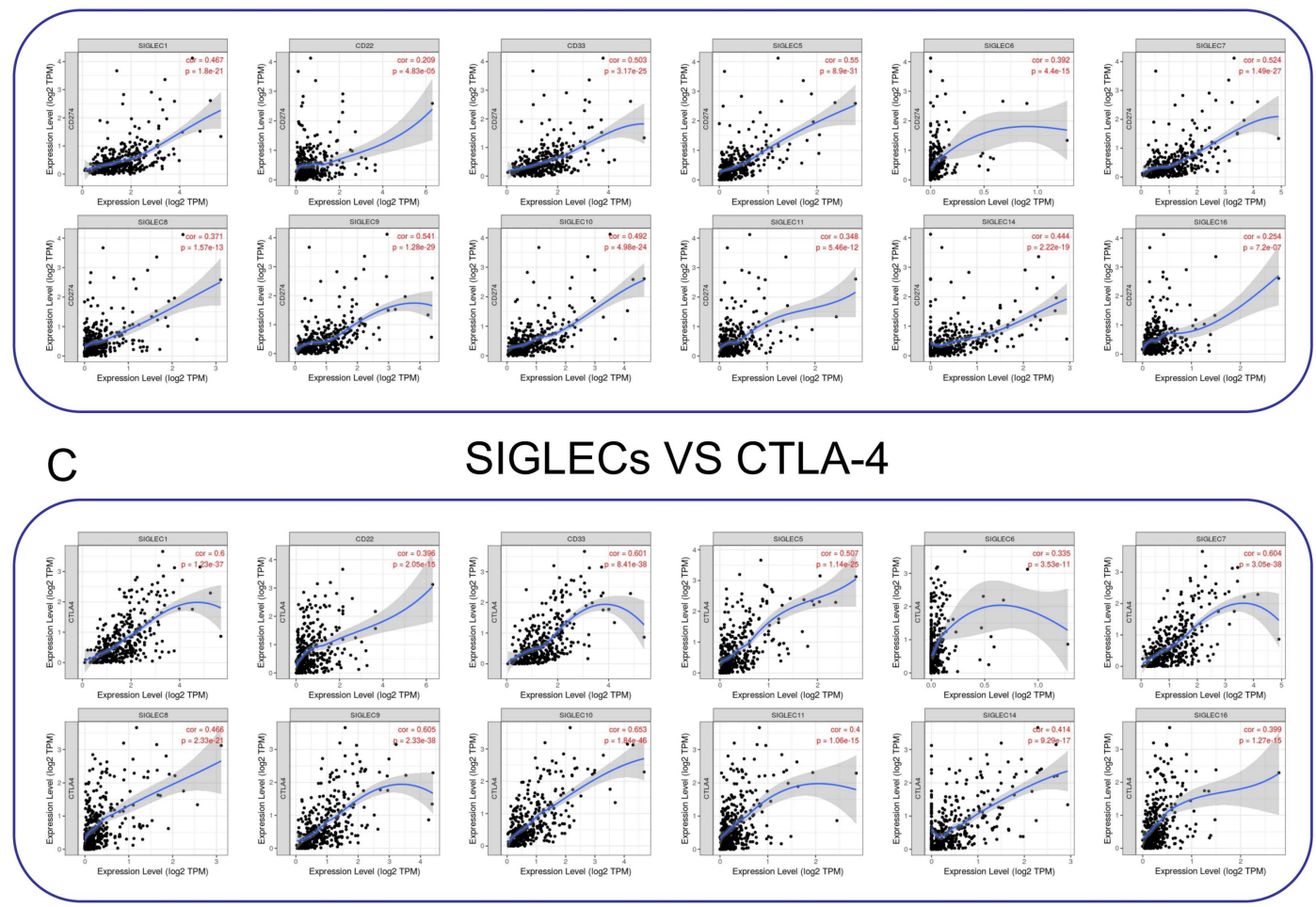

Fig. 6. The correlations were calculated between SIGELCs family and immune genes of PD-1, PD-L1 and CTLA4. (A) The correlations between gene expressions of SIGLECs family and PD-1. (B) The correlations between gene expressions of SIGLECs family and PD-L1. (C) The correlations between gene expressions of SIGLECs family and CTLA-4.

\section{Correlation between protein and mRNA expression of SIGELCs family genes and} clinicopathological characteristics of HCC patients

We downloaded TCGA data through UALCAN,GEPIA and TISIDB and analyzed the relationship between mRNA expression of SIGLECs family members and clinicopathological characteristics (including tumor pathological grade and individual stage) in patients with HCC. The mRNA expression level of SIGLECs family members was significantly correlated with cancer stage. The more advanced the tumor stage, the lower the mRNA expression level of SIGLECs. Similarly, the higher the malignant degree of the tumor grade, the lower the mRNA expression level of SIGLECs(Fig. 7-8). 
In general, the expression of mRNA in part members of SIGLECs was correlated with the clinicopathological characteristics of patients with hepatocellular carcinoma.
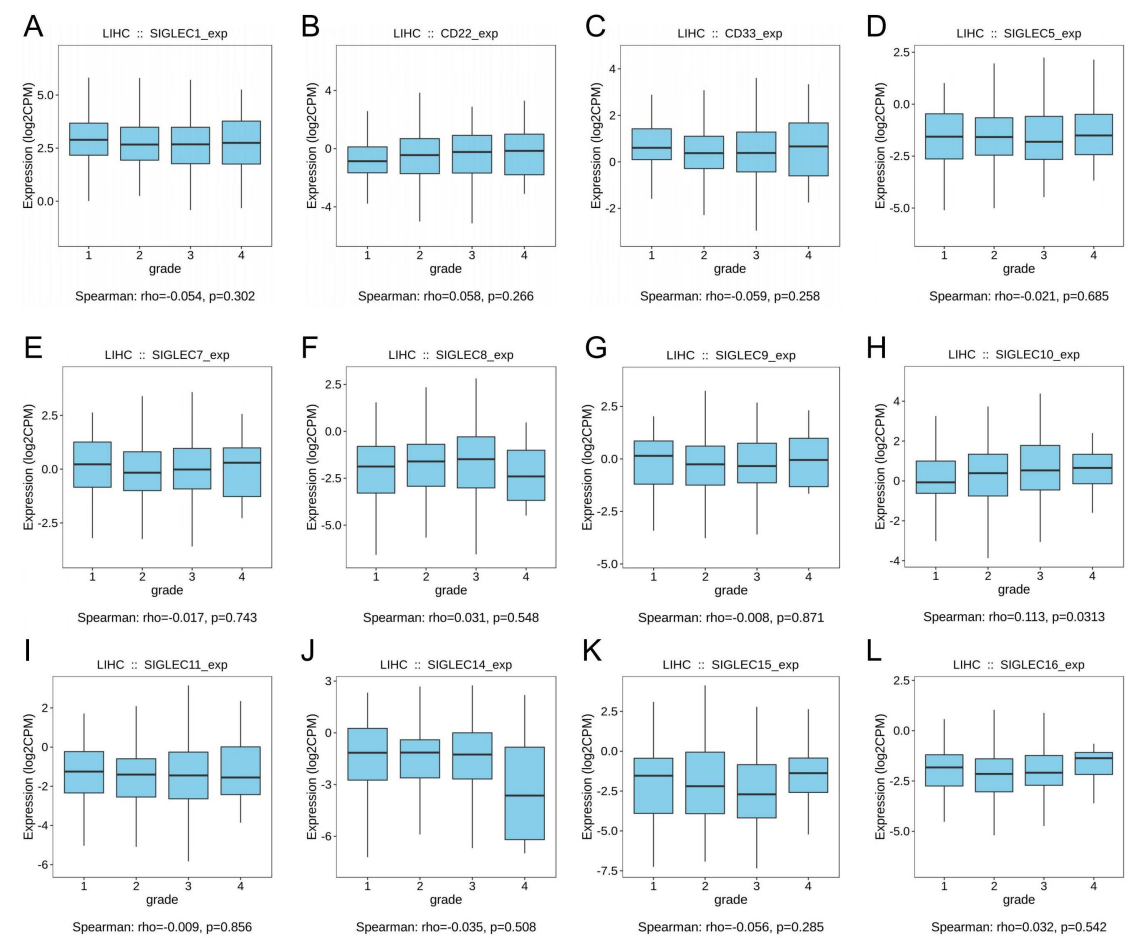

Fig. 7. Association of mRNA expression of SIGLECs family genes with tumor grade of HCC patients. Box figure showing the mRNA expression of SIGLECs family genes in HCC patients in grade 1, 2, 3 or 4 (A-L). 

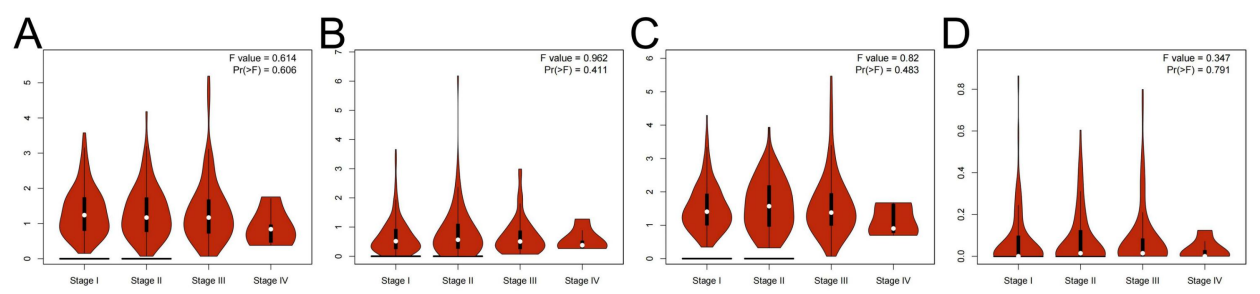

$\mathrm{E}$

$\mathrm{F}$

G

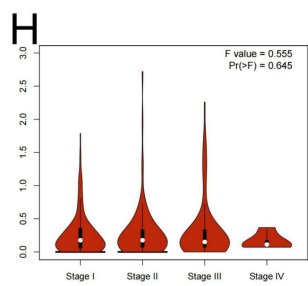

I
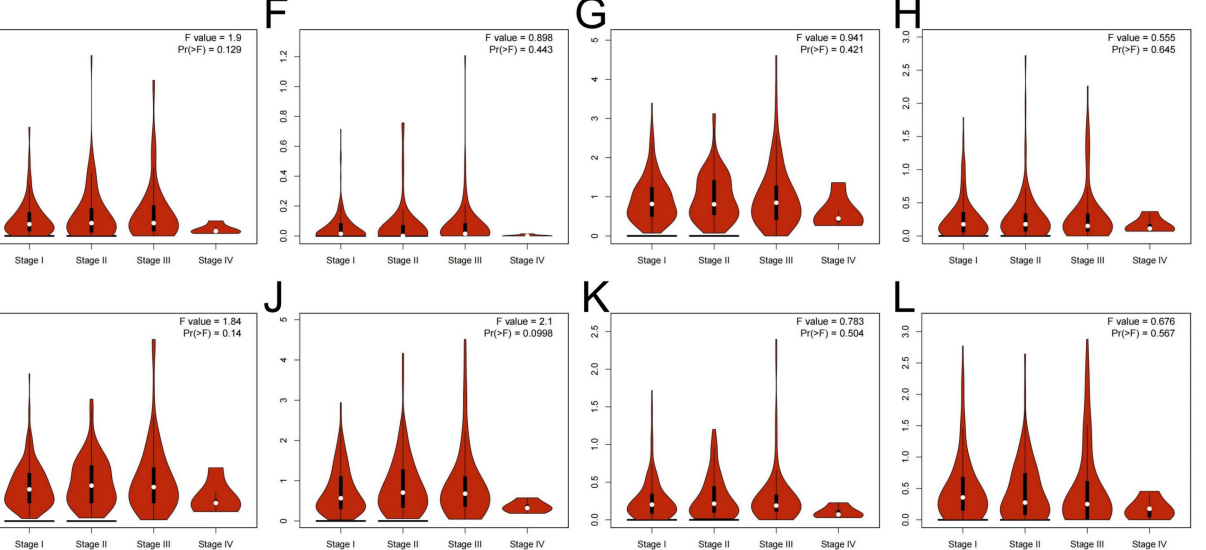

$\mathrm{M}$

$\mathrm{N}$

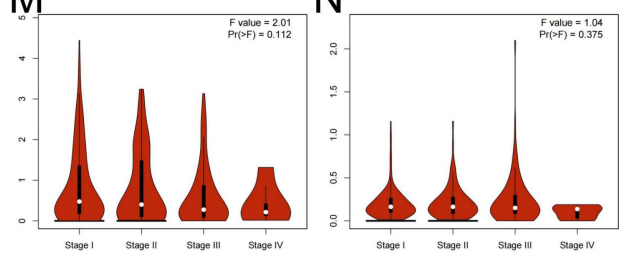

Fig. 8. Association of mRNA expression of SIGLECs family genes with cancer stage of HCC patients. Violin plot showing the mRNA expression of SIGLECs family genes in HCC patients in cancer stages 1, 2, 3 or 4 $(\mathrm{A}-\mathrm{N}) . * \mathbf{p}<0.05 ; * * \mathbf{p}<0.01 ; * * \mathrm{p}<0.001$.

\section{Mutation of SIGLECs family members in hepatocellular carcinoma and its effect on prognosis}

As shown in Figure 9A, we explored the frequency and type of SIGLECs family mutations in 8 data sets of hepatocellular carcinoma (containing 1507 samples) through cBioPortal. Alteration Frenquency in TCGA-Firehose Legacy,TCGA-PanCancer Atlas,INSERM-Nat Genet 2015,AMC-Hepatology is $11.9 \%, 11.7 \%, 8 \%, 5.2 \%, 2.1 \%$, respectively(Fig. 9).

The mutation of SIGLECs family genes was remarkably associated with the prognosis of HCC patients, and the OS, DSS of SIGLECs mutation group was significantly shortened.

From the overall survival analysis, there is a significant connection between the mutation of SIGLECs family and the poor prognosis of HCC patients. The normal expression of SIGLECs family members is beneficial to HCC patients. 


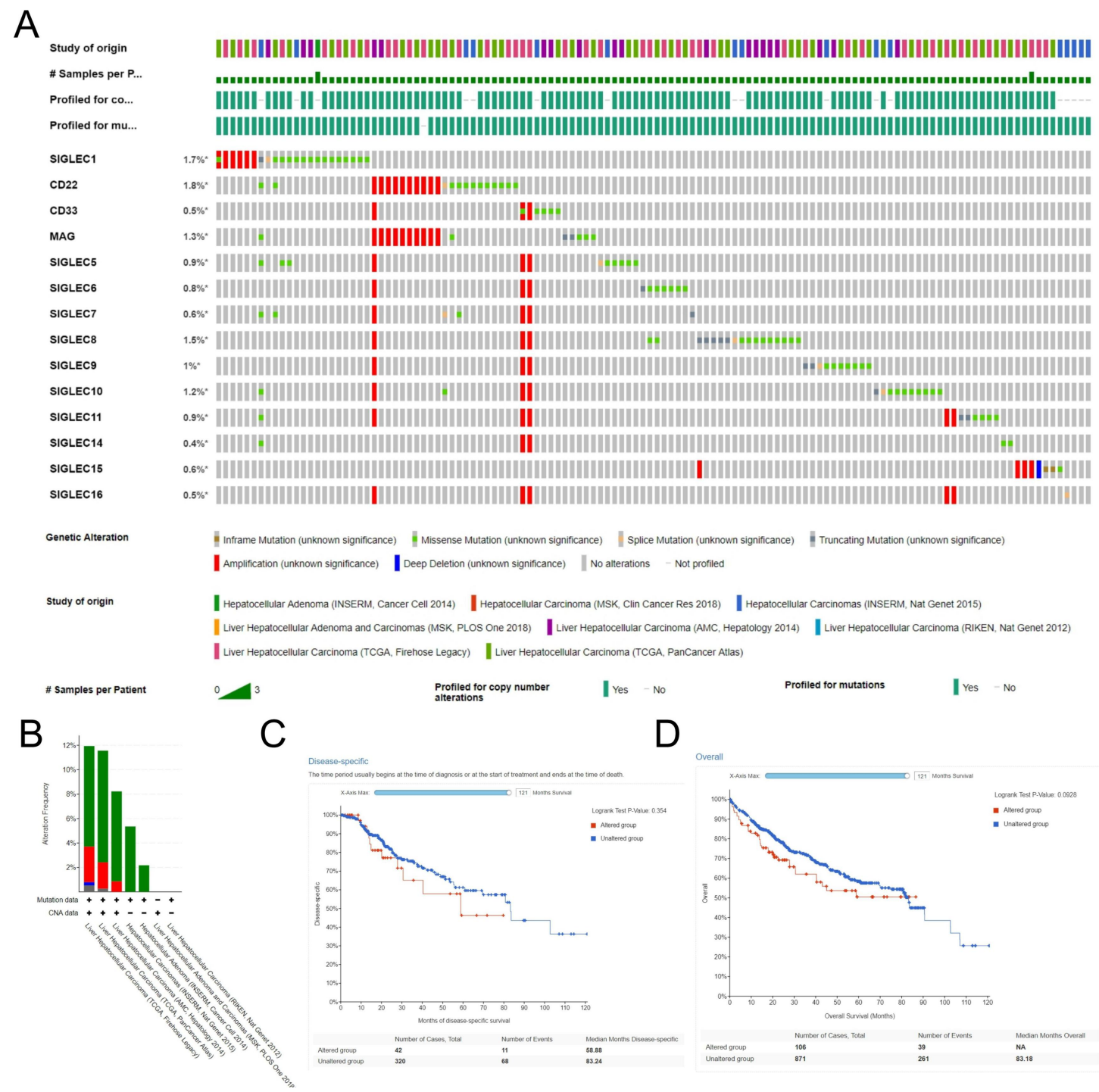

Fig. 9. The frequency and type of SIGLECs family mutations in 8 data sets of HCC (cBioPortal). (A) An overview of SIGLECs mutations. (B) Alteration Frequency of SIGLECs; (C) The Disease Specific Survival of SIGLECs mutation group was significantly shortened; (D) The Overall Survival of SIGLECs mutation group was significantly shortened.

The value of SIGLECs family member mRNA expression in predicting the efficacy of sorafenib in HCC patients

First, we used Kaplan-Meier Plotter to analyze the relationship between mRNA expression of SIGLECs family members and the prognosis of HCC patients. Lower mRNA expression of SIGLECs were significantly associated with shorter RFS and OFS of HCC patients (Fig. 10).

Since after more than ten years of study of sorafenib, the prognosis or predictors of HCC patients' response to the drug have not been verified, we performed an analysis of the expression of SIGLECs family in HCC patients treated with sorafenib. Intriguingly, we were surprised to find that the difference in the expression of SIGLECs family has a far greater impact on the prognosis of HCC patients treated with sorafenib. The high expression of SIGLECs family is significantly associated with the better prognosis of HCC patients (Fig. 11).

These results indicate that differences in gene expression of SIGLECs family will affect the 
progression and prognosis of HCC patients treated with sorafenib. In the future, the SIGLECs family may become a predictive index for the efficacy evaluation of sorafenib, which is of great significance for the prognosis evaluation of HCC patients treated with sorafenib.

A

RFS

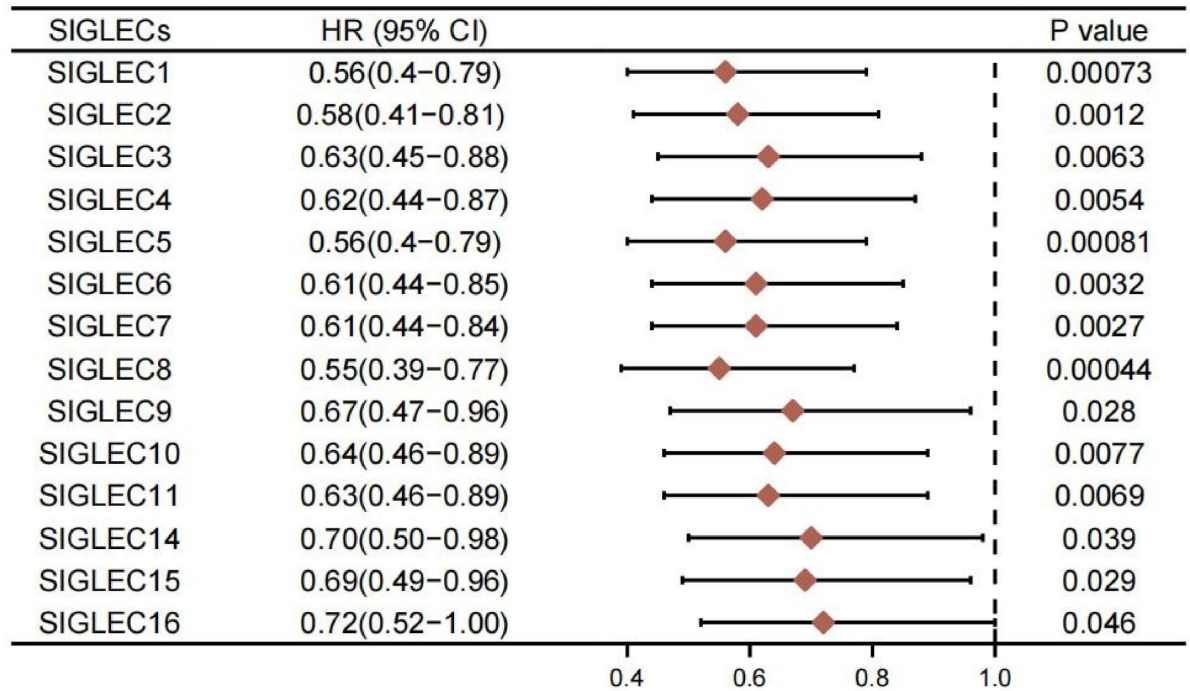

B

PFS

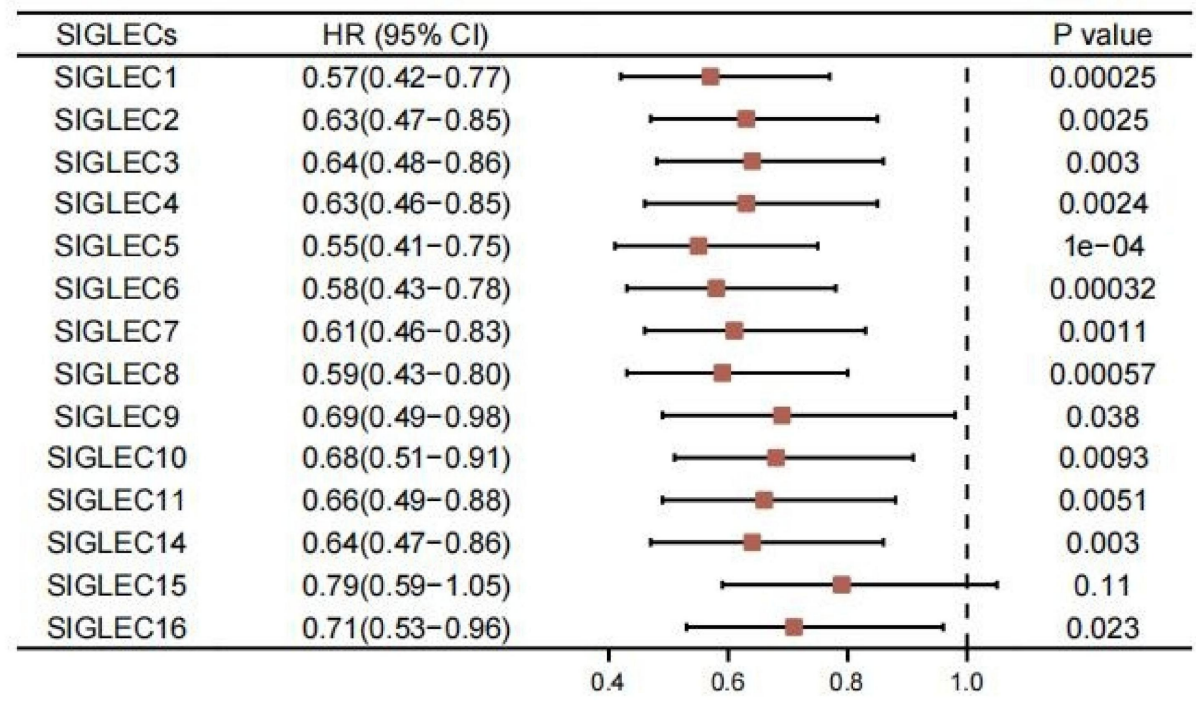

Fig. 10. Forest plots of HRs for RFS and PFS with SIGLECs expression. 

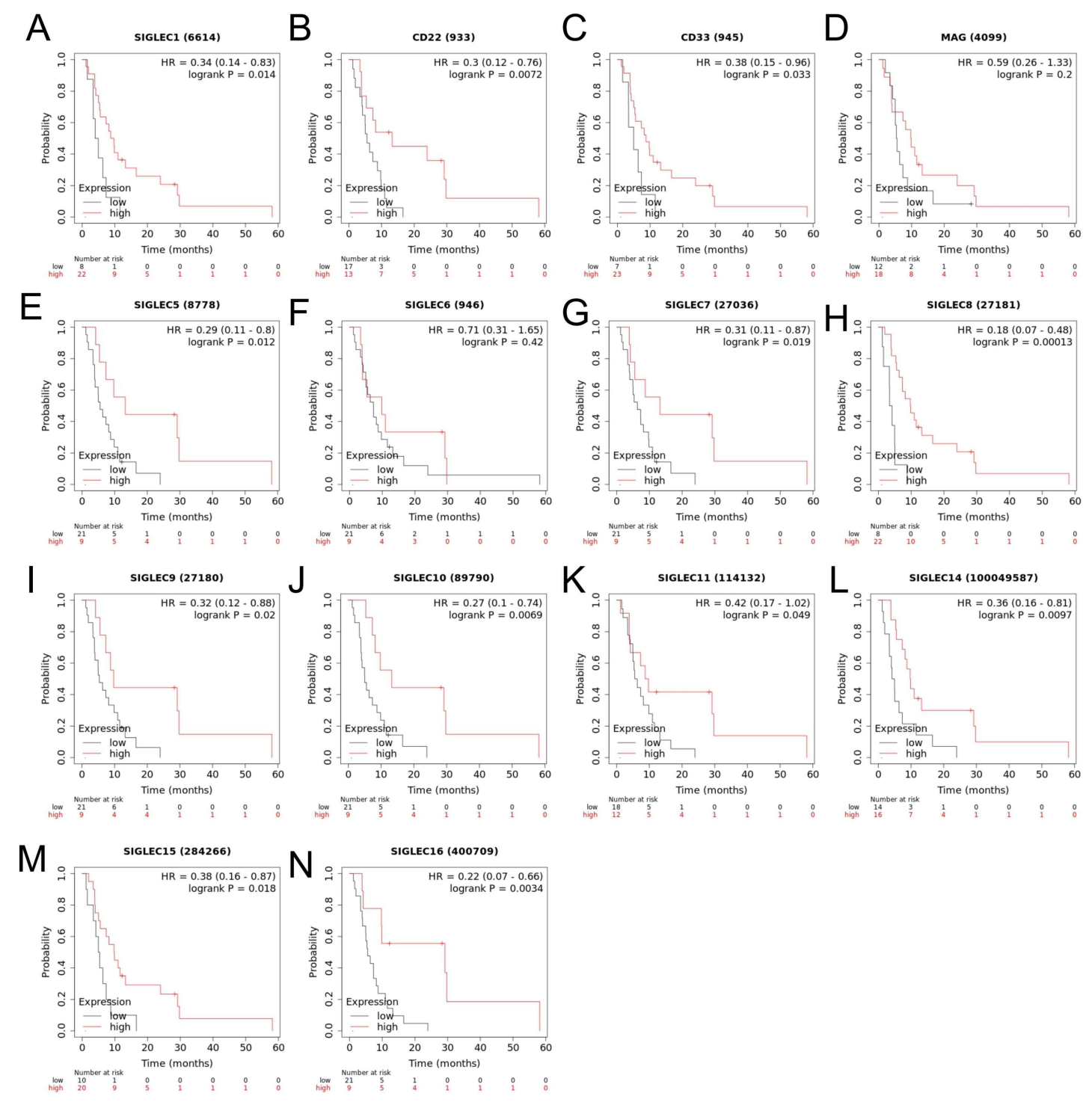

Fig. 11. The effect of SIGLECs expression on survival and prognosis in HCC patients treated with sorafenib.

\section{Discussion}

HCC is a deadly kind of cancer with poor prognosis, which causes higher mortality all over the world, with an increasing number of cases every year. ${ }^{26,27}$ It is possible that the early stages of HCC could be curative. However, the diagnose of early-stage HCC is hard. Because obvious signs are lacking in the early stage of HCC, and available diagnostic biomarkers for HCC are deficient. ${ }^{28}$ Sorafenib has been proven to prolong the survival period of end-stage HCC patients by only several months. ${ }^{29}$ And immunotherapy for HCC is still in its infancy compared to other tumours. ${ }^{30}$ Such kind situation of HCC tratment is far from perfect. Therefore, it is imperative to explore useful targets for the treatment.

The roles of SIGLECs in the regulation of immune cell function in infectious diseases and cancer were already described by Macauley et al. ${ }^{31}$ More recently, a report proposed that SIGLEC family genes conveyed by tumor cells were related to carcinogenesis. ${ }^{13}$ The immune cell infiltration have been confirmed to play crucial functions in HCC progression. ${ }^{32}$ By evaluating the 
correlation between SIGLECs expression with tumor-infiltrating lymphocytes, we found a tight correlation between SIGLECs with immune cell infiltration. Barkal et al informed that CD24 is highly expressed and relates with the inhibitory receptor Siglec10, which is expressed by tumor-associated macrophages to help immune evasion in breast cancer and ovarian cancer. ${ }^{33}$ And it has been informed that Siglec9 is involved in innate immune response to cancer. ${ }^{34}$ There are many studies on the relationship between SIGLECs family and the prognosis of patients with malignant tumor. Kensuke et al showed the relationship between Siglec-7 and CRC prognosis, they found that the expression of Siglec-7 in macrophages may become a novel prognostic biomarker for the efficacy of immunotherapy against metastatic CRC. ${ }^{11}$

Our study showed that mRNA expressions of majority SIGLECs family genes were remarkably down-regulated in HCC tissues from the TCGA and GEO database. Besides, similar results were found by HPA in protein expressions. With further research, we found that proteins including TNR, PTPN11, PLP1, CD19, PSG1, PSG2, and PSG7 related with SIGLECs family genes through PPI network analysis by GeneMANIA. Our findings suggest that the functional effect of SIGLECs mainly include biological adhesion, neutrophil activation, primary cell adhesion. These results are constant with the molecular pathways implicated in HCC carcinogenesis.Subsequently, we analyzed the association of SIGLECs family members expression with clinicopathological factors of HCC patients. The result shows a tendency that the lower the expression of SIGLECs family genes, the worse the stage of tumor and grade in HCC patients. And according to the findings of prognostic analysis ,the expressions of SIGELCs family is remarkably correlated with the better prognosis of HCC patients undergoing sorafenib.

According to present situation, combination plans involving sorafenib and immunotherapy might be a promising method and is currently getting attention in HCC treatment. It is now clear that siglec family members play a comprehensive function in regulating natural immunity. Therefore we explored dual effect of SIGLECs family to overcome the current difficulties of HCC therapy.

\section{Conclusions}

In an overall view, our findings play an significant character in the study of prognostic markers and anti-liver cancer therapeutic targets for members of the SIGLECs family, Our study also provides an important theoretical basis for SIGLECs as an index for predicting the efficacy of sorafenib in the treatment of $\mathrm{HCC}$ in the future.

\section{Ethics approval and consent to participate}

Not applicable.

\section{Consent for publication}

Not applicable.

\section{Availability of data and material}

The datasets during and/or analyzed during the current study available from the corresponding author on reasonable request.

\section{Competing interests}


The authors declare that they have no competing interests.

\section{Funding}

This work was endorsed by the National Natural Science Foundation of China (No. 81872504; 81372487)

\section{Authors' contributions}

Senbang Yao, Wenjun Chen, and Tingting Chen designed the investigation, and He Zuo, Ziran Bi, Xiuqing Zhang helped to accumulate data. Yanyan Jing, Xiangxiang Yin, and Lulian Pang wrote the manuscript of the paper, and Huaidong Cheng helped to modify and make revisions. All authors contributed to the research and authorized the submitted version.

\section{Acknowledgements}

We acknowledge GEO and TCGA for providing their platforms and contributors for uploading their important data.

\section{References}

[1] Siegel RL, Miller KD, Fuchs HE, Jemal A. Cancer Statistics, 2021. CA Cancer J Clin 2021;71(1):7-33. doi: 10.3322/caac.21654. PMID: 33433946

[2] Zhu $C$, Wang A, Zhang $H$, Wan $X$, Yang $X$, Chen S, et al. Research progress and prospects of markers for liver cancer stem cells. World journal of gastroenterology 2015;21(42):12190-12196. doi: 10.3748/wjg.v21.i42.12190. PMID: 26576103

[3] Sun N, Lee $Y$, Zhang R, Kao R, Teng P, Yang Y, et al. Purification of HCC-specific extracellular vesicles on nanosubstrates for early $\mathrm{HCC}$ detection by digital scoring. Nature communications 2020;11(1):4489. doi: 10.1038/s41467-020-18311-0. PMID: 32895384

[4] Poh W, Wong W, Ong H, Aung M, Lim S, Chua B, et al. Klotho-beta overexpression as a novel target for suppressing proliferation and fibroblast growth factor receptor-4 signaling in hepatocellular carcinoma. Molecular cancer 2012;11:14. doi: 10.1186/1476-4598-11-14. PMID: 22439738

[5] Bayard Q, Meunier L, Peneau C, Renault V, Shinde J, Nault JC, et al. Cyclin A2/E1 activation defines a hepatocellular carcinoma subclass with a rearrangement signature of replication stress. Nat Commun 2018;9(1):5235. doi: 10.1038/s41467-018-07552-9. PMID: 30531861

[6] Marisi G, Cucchetti A, Ulivi P, Canale M, Cabibbo G, Solaini L, et al. Ten years of sorafenib in hepatocellular carcinoma: Are there any predictive and/or prognostic markers? World J Gastroenterol 2018;24(36):4152-4163. doi: 10.3748/wjg.v24.i36.4152. PMID: 30271080

[7] Brunetti O, Gnoni A, Licchetta A, Longo V, Calabrese A, Argentiero A, et al. Predictive and Prognostic Factors in HCC Patients Treated with Sorafenib. Medicina 2019;55(10) doi: 10.3390/medicina55100707. PMID: 31640191

[8] Huang P, Low P, Wang I, Hsu S, Angata T. Soluble Siglec-14 glycan-recognition protein is generated by alternative splicing and suppresses myeloid inflammatory responses. The Journal of biological chemistry 2018;293(51):19645-19658. doi: 10.1074/jbc.RA118.005676. PMID: 30377253

[9] Angata T. Associations of genetic polymorphisms of Siglecs with human diseases. 
Glycobiology 2014;24(9):785-793. doi: 10.1093/glycob/cwu043. PMID: 24841380

[10] Bornhöfft K, Viergutz T, Kühnle A, Galuska S. Nanoparticles Equipped with $\alpha 2,8$-Linked Sialic Acid Chains Inhibit the Release of Neutrophil Extracellular Traps. Nanomaterials 2019;9(4) doi: 10.3390/nano9040610. PMID: 31013834

[11] Yamada K, Hazama S, Suzuki N, Xu M, Nakagami Y, Fujiwara N, et al. Siglec-7 is a predictive biomarker for the efficacy of cancer vaccination against metastatic colorectal cancer. Oncology letters 2021;21(1):10. doi: 10.3892/ol.2020.12271. PMID: 33240416

[12] Wang J, Sun J, Liu L, Flies D, Nie X, Toki M, et al. Siglec-15 as an immune suppressor and potential target for normalization cancer immunotherapy. Nature medicine 2019;25(4):656-666. doi: 10.1038/s41591-019-0374-x. PMID: 30833750

[13] Chen Z, Yu M, Guo L, Zhang B, Liu S, Zhang W, et al. Tumor Derived SIGLEC Family Genes May Play Roles in Tumor Genesis, Progression, and Immune Microenvironment Regulation. Front Oncol 2020;10:586820. doi: 10.3389/fonc.2020.586820. PMID: 33240817

[14] Tomczak K, Czerwińska P, Wiznerowicz M. The Cancer Genome Atlas (TCGA): an immeasurable source of knowledge. Contemporary oncology 2015;19:A68-77. doi: 10.5114/wo.2014.47136. PMID: 25691825

[15] Tang Z, Li C, Kang B, Gao G, Li C, Zhang Z. GEPIA: a web server for cancer and normal gene expression profiling and interactive analyses. Nucleic acids research 2017;45:W98-W102. doi: 10.1093/nar/gkx247. PMID: 28407145

[16] Chandrashekar D, Bashel B, Balasubramanya S, Creighton C, Ponce-Rodriguez I, Chakravarthi B, et al. UALCAN: A Portal for Facilitating Tumor Subgroup Gene Expression and Survival Analyses. Neoplasia 2017;19(8):649-658. doi: 10.1016/j.neo.2017.05.002. PMID: 28732212

[17] Barrett T, Wilhite S, Ledoux P, Evangelista C, Kim I, Tomashevsky M, et al. NCBI GEO: archive for functional genomics data sets--update. Nucleic acids research 2013;41:D991-995. doi: 10.1093/nar/gks1193. PMID: 23193258

[18] Roessler S, Jia H, Budhu A, Forgues M, Ye Q, Lee J, et al. A unique metastasis gene signature enables prediction of tumor relapse in early-stage hepatocellular carcinoma patients. Cancer research 2010;70(24):10202-10212. doi: 10.1158/0008-5472.Can-10-2607. PMID: 21159642

[19] Burchard J, Zhang C, Liu A, Poon R, Lee N, Wong K, et al. microRNA-122 as a regulator of mitochondrial metabolic gene network in hepatocellular carcinoma. Molecular systems biology 2010;6:402. doi: 10.1038/msb.2010.58. PMID: 20739924

[20] Zhang J, Baran J, Cros A, Guberman J, Haider S, Hsu J, et al. International Cancer Genome Consortium Data Portal--a one-stop shop for cancer genomics data. Database : the journal of biological databases curation 2011;2011:bar026. doi: 10.1093/database/bar026. PMID: 21930502

[21] Thul P, Ákesson L, Wiking M, Mahdessian D, Geladaki A, Ait Blal H, et al. A subcellular map of the human proteome. Science 2017;356(6340) doi: 10.1126/science.aal3321. PMID: 28495876

[22] Warde-Farley D, Donaldson S, Comes O, Zuberi K, Badrawi R, Chao P, et al. The GeneMANIA prediction server: biological network integration for gene prioritization and predicting gene function. Nucleic acids research 2010;38:W214-220. doi: 10.1093/nar/gkq537. 
PMID: 20576703

[23] Li T, Fan J, Wang B, Traugh N, Chen Q, Liu J, et al. TIMER: A Web Server for Comprehensive Analysis of Tumor-Infiltrating Immune Cells. Cancer research 2017;77(21):e108-e110. doi: 10.1158/0008-5472.Can-17-0307. PMID: 29092952

[24] Cerami E, Gao J, Dogrusoz U, Gross B, Sumer S, Aksoy B, et al. The cBio cancer genomics portal: an open platform for exploring multidimensional cancer genomics data. Cancer discovery 2012;2(5):401-404. doi: 10.1158/2159-8290.Cd-12-0095. PMID: 22588877

[25] Nagy Á, Munkácsy G, Győrffy B. Pancancer survival analysis of cancer hallmark genes. Scientific reports 2021;11(1):6047. doi: 10.1038/s41598-021-84787-5. PMID: 33723286

[26] Asrani S, Devarbhavi H, Eaton J, Kamath P. Burden of liver diseases in the world. Journal of hepatology 2019;70(1):151-171. doi: 10.1016/j.jhep.2018.09.014. PMID: 30266282

[27] Sia D, Villanueva A, Friedman S, Llovet J. Liver Cancer Cell of Origin, Molecular Class, and Effects on Patient Prognosis. Gastroenterology 2017;152(4):745-761. doi: 10.1053/j.gastro.2016.11.048. PMID: 28043904

[28] Jiang Y, Tie C, Wang Y, Bian D, Liu M, Wang T, et al. Upregulation of Serum Sphingosine (d18:1)-1-P Potentially Contributes to Distinguish HCC Including AFP-Negative HCC From Cirrhosis. Frontiers in oncology 2020;10:1759. doi: 10.3389/fonc.2020.01759. PMID: 33014866

[29] Xia J, Ozaki I, Matsuhashi S, Kuwashiro T, Takahashi H, Anzai K, et al. Mechanisms of PKC-Mediated Enhancement of HIF-1 $\alpha$ Activity and its Inhibition by Vitamin K2 in Hepatocellular Carcinoma Cells. International journal of molecular sciences 2019;20(5) doi: 10.3390/ijms20051022. PMID: 30813635

[30] Johnston M, Khakoo S. Immunotherapy for hepatocellular carcinoma: Current and future. World journal of gastroenterology 2019;25(24):2977-2989. doi: 10.3748/wjg.v25.i24.2977. PMID: 31293335

[31] Macauley M, Crocker P, Paulson J. Siglec-mediated regulation of immune cell function in disease. Nature reviews Immunology 2014;14(10):653-666. doi: 10.1038/nri3737. PMID: 25234143

[32] Yang C, Huang X, Liu Z, Qin W, Wang C. Metabolism-associated molecular classification of hepatocellular carcinoma. Molecular oncology 2020;14(4):896-913. doi: 10.1002/1878-0261.12639. PMID: 31955511

[33] Barkal A, Brewer R, Markovic M, Kowarsky M, Barkal S, Zaro B, et al. CD24 signalling through macrophage Siglec-10 is a target for cancer immunotherapy. Nature 2019;572(7769):392-396. doi: 10.1038/s41586-019-1456-0. PMID: 31367043

[34] Hsu Y, Hsu F, Chiang M, Tsai D, Li F, Angata T, et al. Siglec-E retards atherosclerosis by inhibiting CD36-mediated foam cell formation. Journal of biomedical science 2021;28(1):5. doi: 10.1186/s12929-020-00698-z. PMID: 33397354 